\title{
Strange quark momentum fraction from overlap fermion
}

\section{Mingyang Sun*}

Department of Physics and Astronomy, University of Kentucky, Lexington, KY 40506

E-mail: mingyang. sun@uky.edu

\section{Yi-Bo Yang}

Department of Physics and Astronomy, University of Kentucky, Lexington, KY 40506

E-mail: ybyang@pa.uky.edu

\section{Keh-Fei Liu}

Department of Physics and Astronomy, University of Kentucky, Lexington, KY 40506

E-mail: 1iu@pa.uky.edu

\section{Ming Gong}

Institute of High Energy Physics, Chinese Academy of Sciences, Beijing 100049, China

E-mail: gongming@ihep.ac.cn

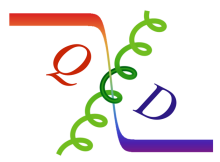

\section{( $\chi Q C D$ Collaboration)}

\begin{abstract}
We present a calculation of $\langle x\rangle_{s}$ for the strange quark in the nucleon. We also report the ratio of the strange $\langle x\rangle$ to that of $u / d$ in the disconnected insertion which will be useful in constraining the global fit of parton distribution functions at small $x$. We adopt overlap fermion action on $2+1$ flavor domain-wall fermion configurations on the $24^{3} \times 64$ lattice with a light sea quark mass which corresponds to $m_{\pi}=330 \mathrm{MeV}$. Smeared grid $Z_{3}$ sources are deployed to calculate the nucleon propagator with low-mode substitution. Even-odd grid sources and time-dilution technique with stochastic noises are used to calculate the high mode contribution to the quark loop. Low mode averaging (LMA) for the quark loop is applied to reduce the statistical error of the disconnected insertion calculation. We find the ratio $\langle x\rangle_{s} /\langle x\rangle_{u / d}^{\mathrm{DI}}=0.78(3)$ in this study.
\end{abstract}

The 32nd International Symposium on Lattice Field Theory

June 22 - June 29, 2014

Columbia University

New York, USA

\footnotetext{
*Speaker.
} 


\section{Introduction}

The understanding of the structure of nucleon has been one of the central issues in hadron physics. For instance, the parton distribution functions (PDFs) have been studied extensively, and the observation of scaling violation in PDFs provides the cradle for the establishment of the fundamental theory, QCD. Yet, there exist many unresolved questions for the structure of the nucleon. Ever since the EMC experiment showed that the proton spin carried by quarks is small[1], large effort has been made in both the experiment and the theoretical frontiers to identify all the contributions to the nucleon spin. Calculating the momentum fraction $\langle x\rangle$ is an integral part of the study of this subject.

However, it has been found that calculating the disconnected insertion contribution to this quantity, which is necessary for the strange momentum fraction, is extremely difficult. Most lattice calculations are done for the connected insertion contribution. So far, only one quenched calculation exists that includes all contributions to the nucleon spin[2]. In this paper, we calculate the strange momentum fraction with the overlap fermion on $2+1$ flavor domain wall fermion configurations with the help of an array of lattice techniques. We also calculate the momentum fraction in the $\mathrm{u} / \mathrm{d}$ channel for the disconnected insertion (DI), and take the ratio between strange and $\mathrm{u} / \mathrm{d}$ channel in DI. In the end, we compare our result with previous lattice calculations, as well as current global analyses of parton distribution at small $x$.

\section{Formalism and simulation parameters}

The momentum fraction carried by quarks can be obtained by calculating the following quark operator of the QCD energy-momentum tensor in the forward matrix element of the nucleon at finite momentum.

$$
T_{4 i}=-\frac{i}{2}\left(\bar{q} \gamma_{4} \stackrel{\leftrightarrow}{D}_{i} q+\bar{q} \gamma_{i} \stackrel{\leftrightarrow}{D}_{4} q\right)
$$

in which

$$
\gamma_{i} \stackrel{\leftrightarrow}{D}_{j}=\frac{1}{2}\left(\gamma_{i} \vec{D}_{j}-\gamma_{i} \overleftarrow{D}_{j}\right)
$$

With this operator, we calculate the disconnected three-point function

$$
C_{3}\left(t_{1}, t_{2}, \vec{p}\right)=\left\langle P_{N}\left(t_{2}, \vec{p}\right) L_{4 i}\left(t_{1}\right)\right\rangle-C_{2}^{N}\left(t_{2}, \vec{p}\right)\left\langle L_{4 i}\left(t_{1}\right)\right\rangle, \quad t_{1}<t_{2}
$$

in which the angular bracket denotes ensemble average, $P_{N}$ is the nucleon propagator, and $L$ denotes the energy-momentum tensor contracted in a quark loop. The momentum-projected nucleon twopoint function $C_{2}^{N}$ is

$$
C_{2}^{N}\left(t_{2}, \vec{p}\right)=\left\langle P_{N}\left(t_{2}, \vec{p}\right)\right\rangle=\sum_{\vec{x}} e^{-i \vec{p} \cdot \vec{x}}\left\langle 0\left|T\left[\chi\left(\vec{x}, t_{2}\right) \bar{\chi}(0,0)\right]\right| 0\right\rangle
$$

where $\chi(\vec{x}, t)$ is the nucleon interpolation field. Note that we take the quark source to be at the origin $\vec{x}=0, t=0$ in the above expression. In the actual calculation, we take the source to be a smeared grid source with a $Z_{3}$ noise. A diagram representing the three-point function is drawn in figure 1 


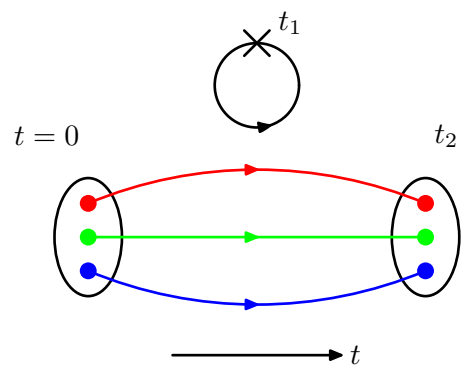

Figure 1: The disconnected insertion

The momentum fraction can then be obtained via the following ratio

$$
-\frac{1}{p} \frac{\operatorname{Tr}\left[\Gamma_{e} C_{3}\left(t_{1}, t_{2}, \vec{p}\right)\right]}{\operatorname{Tr}\left[\Gamma_{e} C_{2}^{N}\left(t_{2}, \vec{p}\right)\right]} \rightarrow\langle x\rangle, \quad t_{2} \gg t_{1} \gg 0
$$

in which $\Gamma_{e}$ is the parity projection operator $\left(1+\gamma_{4}\right) / 2$. To improve signal/noise ratio, we use the sum method[3] by summing the insertion time $t_{1}$ over an appropriate range

$$
-\frac{1}{p} \sum_{t_{1}=1}^{t_{2}-1} \frac{\operatorname{Tr}\left[\Gamma_{e} C_{3}\left(t_{1}, t_{2}, \vec{p}\right)\right]}{\operatorname{Tr}\left[\Gamma_{e} C_{2}^{N}\left(t_{2}, \vec{p}\right)\right]} \rightarrow\langle x\rangle t_{2}+\text { const. }
$$

We use the overlap fermion valence on $N_{f}=2+1$ domain wall fermion configurations by the $\mathrm{RBC} / \mathrm{UKQCD}$ collaboration[ 4 , for which the lattice spacing is $a^{-1}=1.77 \mathrm{GeV}$. The light quark mass is 0.005 in lattice unit, which corresponds to $m_{\pi}=330 \mathrm{MeV}$. For this work, 200 configurations are used. In the valence sector, quark masses $m_{q}=0.00809,0.0135,0.016,0.0203$, and 0.0576 are chosen to be included.

On the choice of momentum for the two-point function, in principle, one may use any finite momentum allowed on the lattice. We choose momenta $p=2 \pi / L a$ and $4 \pi / L a$, and take an appropriate average to acquire a final result.

\section{Lattice techniques}

For the two-point correlation function, several techniques are applied to improve the signal/noise ratio. We use an 8-grid source with gaussian smearing[[] to enhance the signal of the ground state and $Z_{3}$ stochastic noise is used to tie the three-quarks in each smeared source together to have a better overlap with the nucleon. Low mode substitution [6, 7] is also utilized; low mode contribution to the two-point function is calculated exactly. Furthermore, for each configuration, we use 32 sources, each of which is shifted in the time direction. Since at large $t$ where the nucleon effective mass plateau starts to appear, high mode contribution is less important, the inversion of the quark matrix with low-mode deflation is calculated with lower precision to save inversion time. Figure 2 shows the difference in error bars of a point source, grid source, and grid source with low mode substitution (all gaussian-smeared). We should mention that without low-mode substitution, the signal from the grid source is even worse than that from the smeared point source, despite having more statistics (also see [6]). 


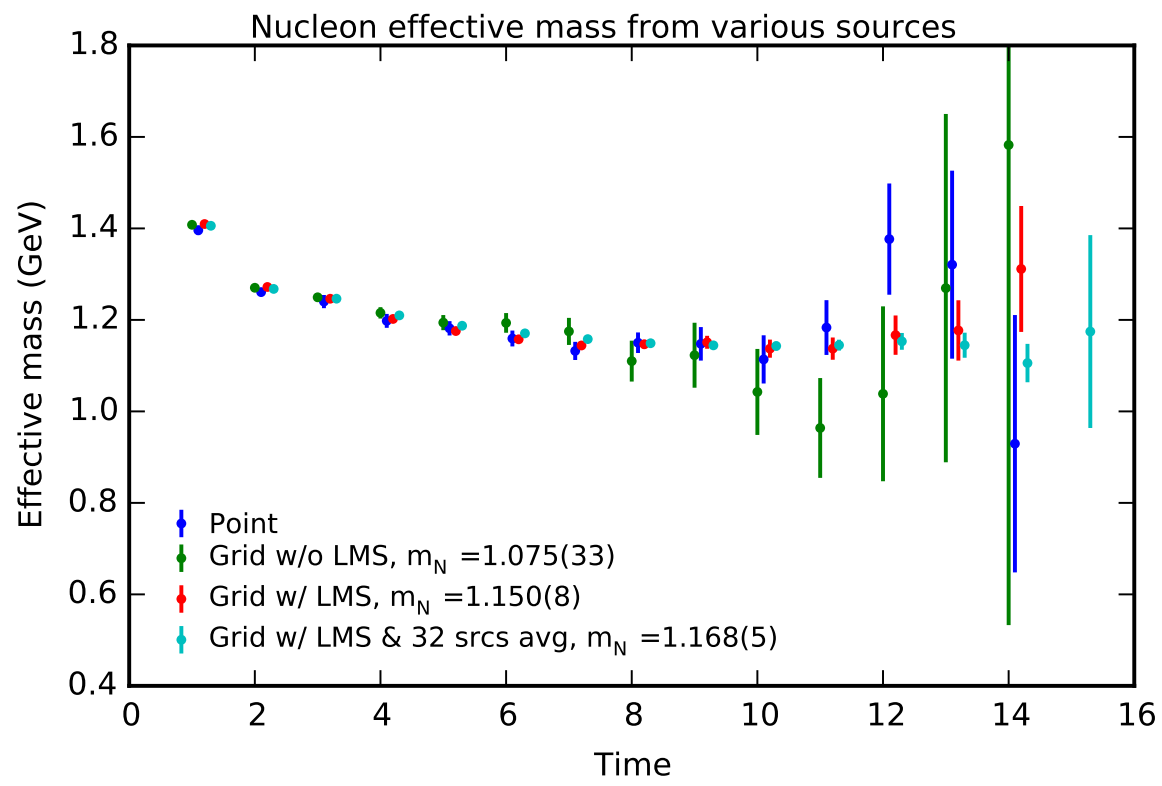

Figure 2: Comparison of nucleon effective mass at $m_{\pi}=330 \mathrm{MeV}$ plotted from different source schemes (as ordered in the legend: a single point, 8-grid without LMS, 8-grid with LMS, and 8-grid with LMS from 32 sources per configuration as described in this paper). In this graph, all sources are gaussian smeared. "Grid w/ LMS \& 32 srcs avg" is used in this work.
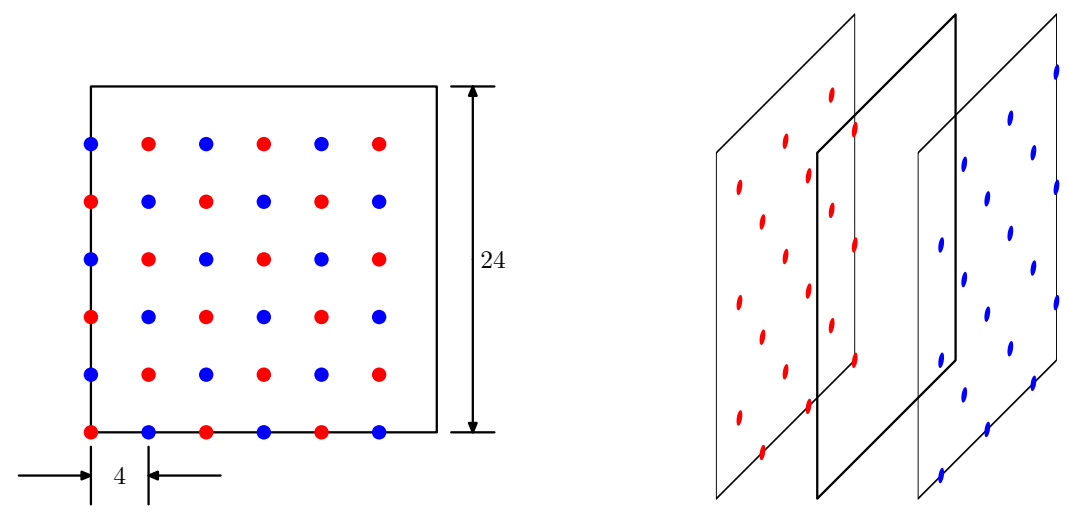

Figure 3: Source scheme for the loop. The right figure shows the grid points on different time slices in each diluted inversion.

For the loop, we use a grid source with $Z_{4}$ noises in both spacial and time direction, and the sources are arranged in a even-odd fashion. For each configuration, 32 sources are used. Among all these sources, the grid is shifted in spacial directions, so that these sources together cover all time slices, and $1 / 4$ of the lattice sites in any given time slice. A sketch of this source scheme is drawn in figure 3. Similar to the two-point functions, we utilize the low mode averaging (LMA) technique [8], and calculate the low mode contribution to the loop exactly without any stochastic noise, and high mode contribution is calculated with lower precision. 


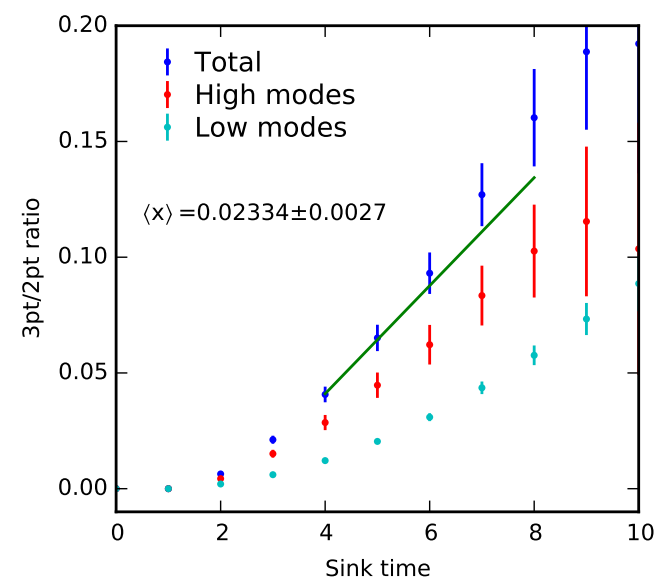

(a) $\langle x\rangle$ slope in the $\mathrm{u} / \mathrm{d}$ channel

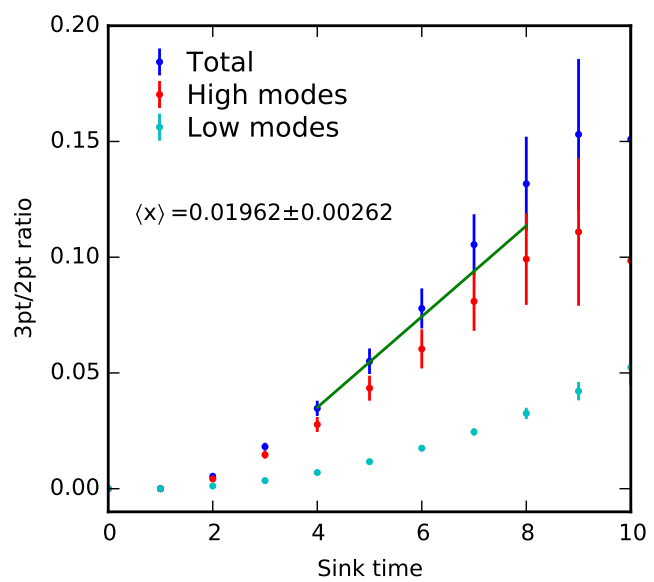

(b) $\langle x\rangle$ slope in the strange channel

Figure 4: 3-point/2-point function ratio in the form of slope as a function of the nucleon sink-source time separation from the sum method.

\section{Results}

In order to improve the signal/noise ratio, we use the sum method to calculate the physical quantities we want, i.e. $\langle x\rangle$, from the 3-point function (eq. 2.5). Figure ta shows the signals in the $\mathrm{u} / \mathrm{d}$ channel, in which both the 2-point function and the loop are with quark mass $0.016\left(m_{\pi}=\right.$ $330 \mathrm{MeV}$ ); figure $4 \mathrm{bb}$ shows the signal in the strange channel, in which the nucleon propagator is calculated with quark mass 0.016 , and the loop with 0.0576 which is at the strange quark mass. All the techniques described above contribute to the resulting good signals. In the $\mathrm{u} / \mathrm{d}$ channel, we are able to reach a clear signal almost 9 sigmas away from zero. Furthermore, contribution from high modes and low modes are also plotted. It is evident that the high modes contribution is significant for $\langle x\rangle$, hence we devote a large amount of computational time in order to control its error.

We show in figure $5\langle x\rangle_{u / d}^{\mathrm{DI}}$ and $\langle x\rangle_{s}$ as a function of $m_{\pi}^{2}$ for several quark masses in the nucleon propagator and the $u / d$ quark loop; while the strange quark mass in the loop is fixed. We then extrapolate to the physical pion mass using the following functional forms respectively

$$
\langle x\rangle_{u d}^{\mathrm{DI}}=a_{1}+a_{2} m_{\pi}^{2}+a_{3} m_{\pi}^{4}, \quad\langle x\rangle_{s}=b_{1}+b_{2} m_{K}^{2} .
$$

in which the $a$ 's and $b$ 's are fitting parameters. Thus we obtain both $\langle x\rangle_{u / d}^{\mathrm{DI}}$ and $\langle x\rangle_{s}$ at the physical point

$$
\langle x\rangle_{u / d}^{\mathrm{DI}}=0.0285(57), \quad\langle x\rangle_{s}=0.0195(26) .
$$

They will be renormalized and matched to the $\overline{M S}$ scheme [9].

In figure 6, we plot the ratio $\langle x\rangle_{s} /\langle x\rangle_{u / d}^{\mathrm{DI}}$ for each quark mass, and take a chiral extrapolation in terms of the linear $m_{\pi}^{2}$. The extrapolated value is found to be

$$
\frac{\langle x\rangle_{s}}{\langle x\rangle_{u / d}^{\mathrm{DI}}}=0.78(3) .
$$




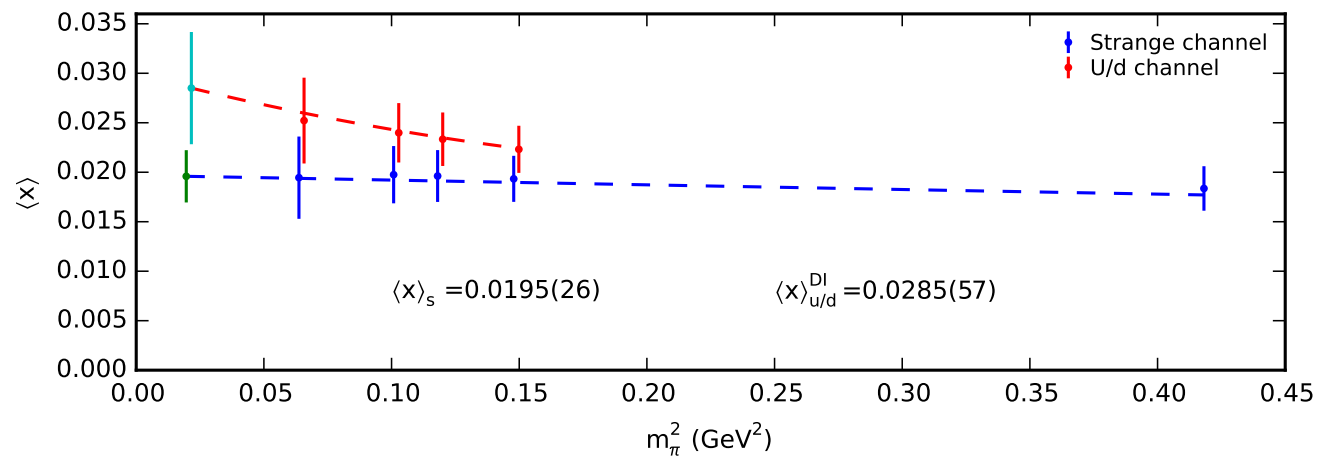

Figure 5: $\langle x\rangle_{u d}$ and $\langle x\rangle_{s}$ extrapolated to the physical value

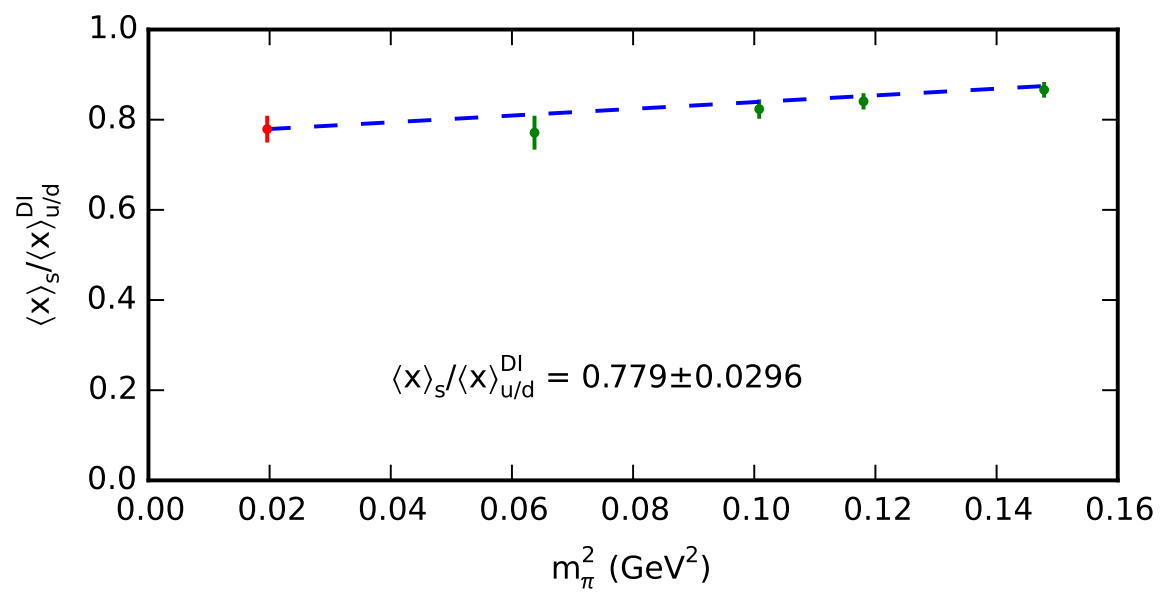

Figure 6: $\langle x\rangle_{s} /\langle x\rangle_{u / d}^{\mathrm{DI}}$ ratio extrapolated to the physical pion mass.

\section{Conclusion and Discussion}

We have studied the momentum fraction carried by quarks in nucleon with overlap fermions on $2+1$ flavors domain wall dynamical fermion configurations. We utilize an array of lattice techniques including low mode substitution and low mode averaging, as well as grid source with $Z_{3}$ and $Z_{4}$ noises to greatly improve the signal of both the nucleon propagator and the quark loop. We find clear signals for $\langle x\rangle_{u / d}^{\mathrm{DI}}$ with a 5 sigma signal and $\langle x\rangle_{s}$ with a 7 sigma signal at the physical pion mass. It is worth noting that our ratio result $\langle x\rangle_{s} /\langle x\rangle_{u / d}^{\mathrm{DI}}=0.78(3)$ is consistent with the global analysis shown in figure 7[10], given that the "disconnected sea" contribution dominates small $x$ region due to its $x^{-1}$ dependence[11, [12], and that the $(s+\bar{s}) /(\bar{u}+\bar{d})$ ratio is relatively flat in small $x$ region. We would also like to point out that our result is in agreement with previous lattice calculations by T. Doi et al. [13] and M. Deka et al. [2]. This ratio can be used to better constrain the global fitting of the parton distribution functions at small $x$. 


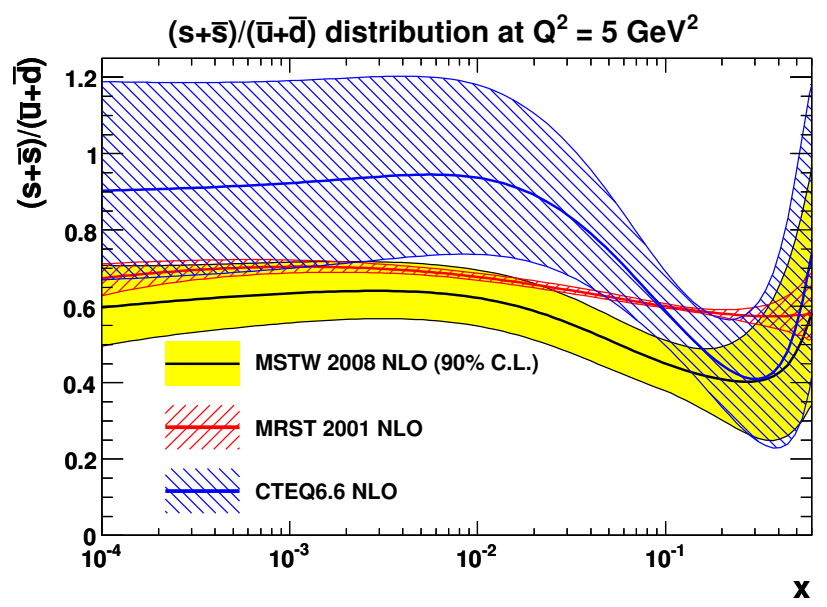

Figure 7: Ratio of $s+\bar{s}$ over $\bar{u}+\bar{d}$ versus $x$ at $Q^{2}=5 \mathrm{GeV}^{2}$ from various recent PDFs.

\section{References}

[1] J. Ashman et al., A measurement of the spin asymmetry and determination of the structure function $\mathrm{g} 1$ in deep inelastic muon-proton scattering, Physics Letters B 206, 364 (1988).

[2] M. Deka et al., Moments of nucleon's parton distribution for the sea and valence quarks from lattice QCD, Phys. Rev. D 79, 094502 (2009).

[3] L. Maiani, G. Martinelli, M. L. Paciello, and B. Taglienti, Scalar densities and baryon mass differences in lattice QCD with Wilson fermions, Nuclear Physics B 293, 420 (1987).

[4] T. Yamazaki et al., Nucleon Axial Charge in (2+1)-Flavor Dynamical-Lattice QCD with Domain-Wall Fermions, Phys. Rev. Lett. 100, 171602 (2008).

[5] C. Alexandrou, S. Güsken, F. Jegerlehner, K. Schilling, and R. Sommer, The static approximation of heavy-light quark systems. A detailed lattice study, Nuclear Physics B 414, 815 (1994).

[6] A. Li et al., Overlap Valence on 2+1 Flavor Domain Wall Fermion Configurations with Deflation and Low-mode Substitution, Phys. Rev. D 82, 114501 (2010), arXiv:1005.5424.

[7] M. Gong et al., Strangeness and charmness content of the nucleon from overlap fermions on 2+1-flavor domain-wall fermion configurations, Phys. Rev. D 88 (2013), arXiv:1304.1194.

[8] J. Foley et al., Practical all-to-all propagators for lattice QCD, Computer Physics Communications 172, 145 (2005), arXiv:hep-lat/0505023.

[9] M. Glatzmaier and K.-F. Liu, Perturbative Renormalization and Mixing of Quark and Glue Energy-Momentum Tensors on the Lattice, arXiv:1403.7211 (2014), arXiv: 1403.7211.

[10] A. D. Martin, W. J. Stirling, R. S. Thorne, and G. Watt, Parton distributions for the LHC, The European Physical Journal C 63, 189 (2009), arXiv: 0901.0002.

[11] K.-F. Liu, W.-C. Chang, H.-Y. Cheng, and J.-C. Peng, Connected-Sea Partons, Phys. Rev. Lett. 109, 252002 (2012).

[12] J. C. Peng, W. C. Chang, H. Y. Cheng, and K. F. Liu, The Flavor Structure of the Nucleon Sea, arXiv:1402.1236 (2014), arXiv: 1402.1236. 
[13] T. Doi et al., Strangeness and glue in the nucleon from lattice QCD, PoS LATTICE2008 (2008), arXiv: 0810.2482. 